\title{
Determining the optimum cell size of digital elevation model for hydrologic application
}

\author{
Arabinda Sharma $^{1, *}$, K N Tiwari ${ }^{2}$ and P B S Bhadoria ${ }^{2}$ \\ ${ }^{1}$ Civil Engineering Department, BRCM College of Engineering \& Technology, Bahal 127 028, \\ Bhiwani, Haryana, India. \\ ${ }^{2}$ Agricultural \& Food Engineering Department, Indian Institute of Technology, \\ Kharagpur-721302, West Bengal, India. \\ *e-mail: arbind_78@rediffmail.com
}

\begin{abstract}
Scale is one of the most important but unsolved issues in various scientific disciplines that deal with spatial data. The arbitrary choice of grid cell size for contour interpolated digital elevation models (DEM) is one of the major sources of uncertainty in the hydrologic modelling process. In this paper, an attempt was made to identify methods for determining an optimum cell size for a contour interpolated DEM in prior to hydrologic modelling. Twenty-meter interval contour lines were used to generate DEMs of five different resolutions, viz., 30, 45, 60, 75, and $90 \mathrm{~m}$ using TOPOGRID algorithm. The obtained DEMs were explored for their intrinsic quality using four different methods, i.e., sink analysis, fractal dimension of derived stream network, entropy measurement and semivariogram modelling. These methods were applied to determine the level artifacts (interpolation error) in DEM surface as well as derived stream network, spatial information content and spatial variability respectively. The results indicated that a $90 \mathrm{~m}$ cell size is sufficient to capture the terrain variability for subsequent hydrologic modelling in the study area. The significance of this research work is that it provides methods which DEM users can apply to select an appropriate DEM cell size in prior to detailed hydrologic modelling.
\end{abstract}

\section{Introduction}

The term scale itself is an ambiguous word with several scientific and dictionary meanings. Scale represented in the form of a basic building unit or grid cell size of digitally mapped data such as digital elevation models (DEM), often linked to degree of detail and precision. Scale is one of the important issues in many branches of spatial sciences (Wilson and Gallant 2000). DEM scale as imposed by grid cell resolution continues to be an important consideration for DEMs used for hydrologic analysis (Wechsler 2007). In most of the spatial projects the cell size is defined arbitrarily or using some heuristic rule without any objectivity. For example, the suitable grid resolution can be estimated by dividing the size of the area with two times the total length of digitized contour. Another heuristic rule to determine the optimum resolution is that the output DEM resolution is $10^{-3}$ of the map scale (Hengl 2006). Nevertheless, such choices of grid cell size are often made arbitrarily and do not necessarily reflect an optimum size for a particular analysis. For instance,

Keywords. DEM; cell size; sink; fractal dimension; entropy; semivariogram. 
a coarse grid may fail to capture the surface variation due to micro-topography, meaning that some peaks and channels might disappear in a raster DEM. On the contrary, if the grid resolution is too fine it might contain more 'noise', suffered from data redundancy and this can burden the storage disk and computer's processing power. Thus, such subjective choice of cell size imparts huge amount of uncertainty in the subsequent modelling.

The DEM constitutes an important spatial data for different branches of earth sciences including hydrology. Although the raster structure of DEM offers numerous opportunities for hydrological application, the issue of scale as imposed by the arbitrary choice of DEM cell size is a significant source of uncertainty. Researchers have investigated the issue of grid cell resolution and its impact on varied application. The literature has established that the DEM grid cell size significantly affects a wide range of hydrological derivatives such as topographic parameters, channel networks and catchment discretization (Florinsky and Kuryakova 2000; Lacroix et al 2002). DEM cell size also affects the spatial prediction of soil attributes (Thompson et al 2001), computation of geomorphic indices (Hancock 2005), computation of soil water content (Kuo et al 1999), and topographic index distribution (Aryal and Bates 2008), river flow and surface inundation simulation (Dutta and Nakayama 2009). Several hydrological modellers reported the DEM cell effect on their modelling outputs. Most notable is the effect of grid size on the output from the popular rainfall-runoff models such as TOPMODEL (Sun et al 2008), the Soil and Water Assessment Tool (Chaubey et al 2005) and the Agricultural Nonpoint Source Pollution (Singh et al 2006). The soil loss estimation using different soil erosion models have also been severely affected by DEM cell size, e.g., CASC2D-SED (Rojas et al 2008), RUSLE (Lee and Lee 2006), WEPP (Zhang et al 2008) and USLE (Wu et al 2005).

All these studies led to identical conclusions that as resolution decreases, derived terrain parameters decrease, and many delicate landscape features are lost. However, as one can understand, it is not enough to model the cell size effects. Indeed, it would be more desirable to find an optimum cell size for a particular analysis or application, if it exists. In spite of several efforts in recent years, scale is still a major unsolved issue in spatial hydrology. Hence, an attempt was made to develop methods to be used to determine an optimum DEM cell size for hydrological application by analyzing the intrinsic nature (sink, fractal dimension, entropy and spatial variability) of contour interpolated DEM in prior to detailed modelling.

\section{Methodology}

\subsection{Study area and DEM preparation}

The Maithon Reservoir Catchment, lying between $23^{\circ} 45^{\prime}-24^{\circ} 34^{\prime} \mathrm{N}$ latitude and $85^{\circ} 25^{\prime}-86^{\circ} 54^{\prime} \mathrm{E}$ longitude, situated in the Jharkhand state of India was selected for this study. The watershed covers an area of $5310 \mathrm{~km}^{2}$ and the altitude varies between 120 and $1340 \mathrm{~m}$ above sea level. In order to explore the effect of terrain complexity on the sink distribution and information content, the study area was divided into three terrain complexity classes (TCC) based on elevation and standard deviation (SD) of slope (in degree) value:

i) TCC-1 (elevation $<220 \mathrm{~m}$ and slope $\mathrm{SD}$ $<1.247)$

ii) TCC-2 $(220 \mathrm{~m} \leqslant$ elevation $\leqslant 350 \mathrm{~m}$ and $1.247 \leqslant$ slope $\mathrm{SD} \leqslant 3.862)$, and

iii) TCC-3 (elevation $>350 \mathrm{~m}$ and slope SD $>3.862)$.

Figure 1 shows the location of the study area and distribution of various TCCs.

The cartographic DEMs which are based on the principle of interpolation of contour lines were used in this study. Contour lines of $20 \mathrm{~m}$ interval digitized from 1:50,000 scale Survey of India topographic maps were used for DEM generation. TOPOGRID command and its subcommands implemented in an ArcInfo GIS version 8.3 were selected for DEM generation because many earlier researches have shown that it preserves the drainage feature more accurately than any other interpolator (Sharma et al 2009). DEMs with cell sizes ranging from 30 to $90 \mathrm{~m}$ at $15 \mathrm{~m}$ interval were interpolated from the contour lines. To search the optimum cell size, the intrinsic nature of DEMs was analyzed for investigated cell size using sink analysis, information content and spatial variability.

\subsection{Sink analysis}

Sinks are the erroneous depressions in terrain surface created by interpolators due to incompatibility in the data density and level of spatialization. Two different characteristics of sinks, namely, number of sinks and total area of sinks were considered in sink analysis. Even a single cell sink can disrupt the hydrologic continuity of the surface and make the DEM unsuitable for subsequent hydrologic analysis which requires a surface with undisrupted flow. The total surface area of sinks in a DEM indicates the amount of alteration that would be brought to original DEM by any sink removal algorithm. The numbers of sink in different DEMs were determined using grid command sink of ArcInfo software version 8.3. The total surface area of sinks 


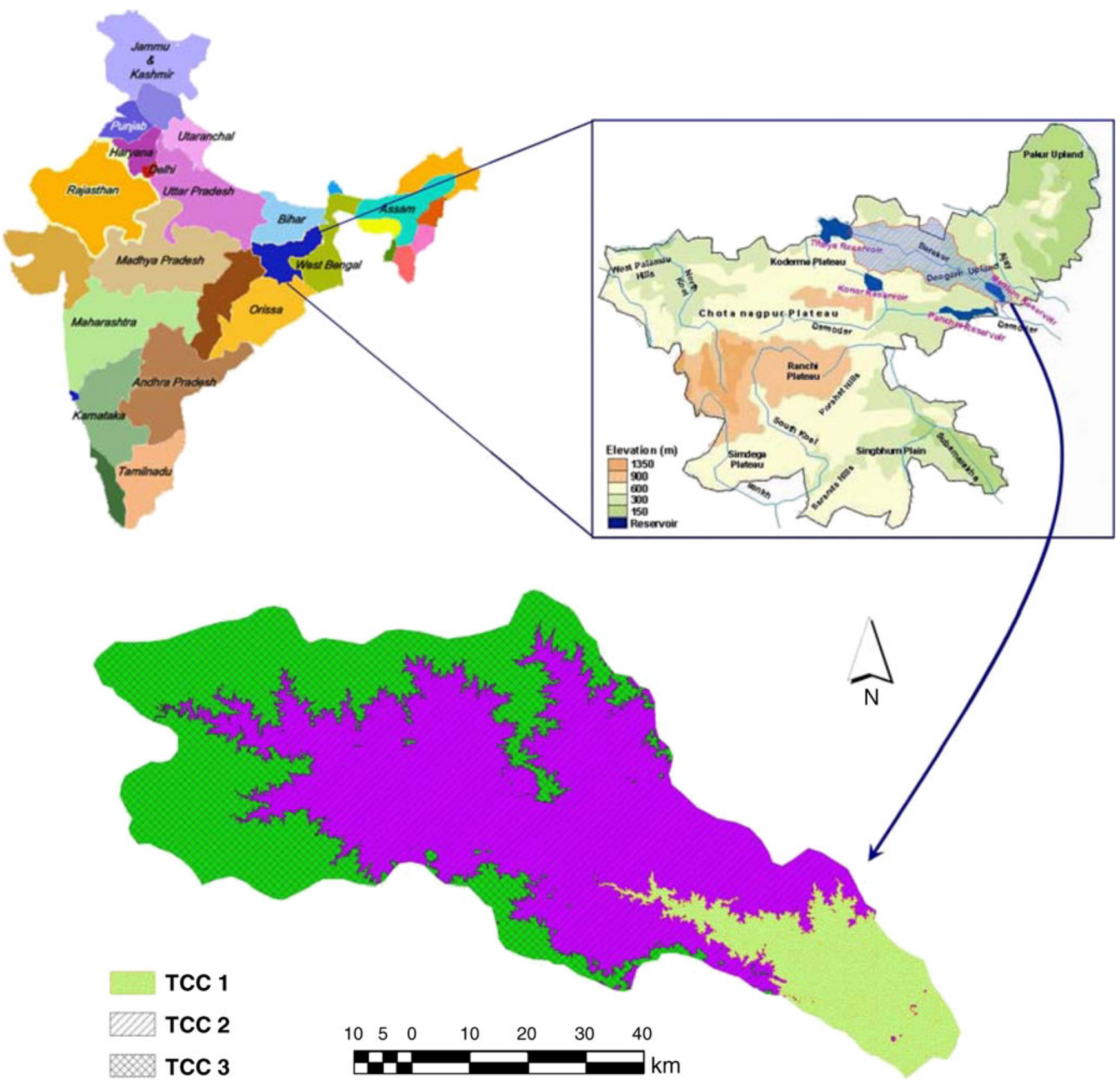

Figure 1. Map of the study area showing the distribution of various terrain complexity classes.

was calculated by subtracting the sink-filled DEMs from the original DEMs. The sink-filled DEMs were produced by using grid command fill of ArcInfo software version 8.3.

\subsection{Fractal dimension}

According to fractal geometry (Mandelbrot 1982), several natural objects behave as fractal bodies which display self-similarity, i.e., if split into parts, each of which is (at least approximately) a reducedsize copy of the whole. Fractal analysis can be used effectively to determine optimum spatial resolution of DEM (Sun et al 2006; Abedini and Shaghaghian
2009). Fractal dimension (a scaling index of fractal nature of an object) was used as characterized fractal bodies. Stream networks exhibit fractal characteristics. The fractal dimension $(D)$ value of DEM-extracted stream networks was used as an indicator of DEM quality. The fractal dimension reveals the production artifacts or anomalies or excessive smoothing in the DEM. The box count method (Chen and Gong 2004) was used in this study to compute the fractal dimension. The counting proceeds, step by step, follow an iteration procedure. At each iteration step, the method counts the number of boxes of size $\varepsilon$ required to cover all 
the stream pixels. The fractal dimension can be expressed as:

$$
D=\frac{\log N}{\log \varepsilon} .
$$

The size of the box is enlarged step by step. Usually, the value set of $\varepsilon$ follows a power of 2 . By doing that, we artificially changed the level of analysis or elementary unit of measurement of stream network. A graph can be plotted with Yaxis corresponding to the logarithms of number of counted stream pixels $(N)$ and the $\mathrm{X}$-axis corresponding to the logarithms of size of box. The slope of the plot provides the fractal dimension $(D)$ of the object.

Any deviation in fractal dimension of extracted stream network from that of a reference stream network (blue lines digitized from topographic maps) can be considered as artifact. Stream network were delineated from depressionless DEMs of different resolutions using the accumulation threshold method. The thresholds for stream extraction were selected empirically by visually comparing stream density of derived stream network and that of the reference stream network. The threshold for other DEMs were selected as inverse proportion of their cell size, i.e., the threshold for $30 \mathrm{~m}$ DEM (4500 pixels) would be nine times as that of $90 \mathrm{~m}$ DEM (500 pixels) and so on. The proportionate selection of accumulation thresholds avoided the chance of overdensification of stream network for finer resolution DEMs.

\subsection{Information content-entropy}

The concept of entropy or theory of information content (Shannon and Weaver 1949) was used to measure spatial information in interpolated DEMs. Entropy of an interpolated DEM $(H)$ can be calculated using equation (2).

$$
H=-\sum_{i=1}^{m}\left(P_{i} \times \log _{2} P_{i}\right),
$$

where $P_{i}$ is the probability of a cell being classified as class type ' $i$ ' and $m$ is the number of classes. With the increase in information content, entropy increases. Entropy will be lowest when the prediction of elevation information for a surface is ensured or there is no spatial variability such as in flat area. In contrast, maximum entropy will be obtained for equally probable elevations, i.e., a surface with uniform slope or one with high spatial variability. When a DEM either gets oversampled (small cell size) or undersampled (large cell size), the spatial variation in elevation value is low due to the redundant elevation value or loss of micro relief structure respectively. In both the cases, the entropy will be low.

\subsection{Spatial variability - semivariogram modelling}

Spatial variability of the resultant DEMs were measured using mean local variance (MLV), semivariogram and semivariance at a lag distance of one pixel. The mean local variance is defined as the average of the variances calculated within a given neighbourhood. In this study, a sliding window of $900 \times 900 \mathrm{~m}$ size was used to measure the local standard deviation. The obtained grid was then squared and subsequently a global mean was derived for each DEM of different resolution. The logic behind using MLV is that, if the spatial resolution is considerably finer than the objects in the area, most of the measurements will be highly correlated with their neighbours and thus the local variance will be low. On the other hand, if the objects studied approximate the size of the resolution, the values tend to be different from each other and therefore the local variance increases.

The spatial variability of interpolated DEMs was also compared using geostatistical method. Semivariogram was used to explore the spatial structure of each DEM dataset by relating the semivariance between two sample points to the distance that separates them. Semivariogram modelling was earlier used by researchers to identify the optimum cell size for various spatial data (Atkinson and Curran 1995; Liu et al 2009). Semivariance can be measured using equation (3) (Goovaerts 1999).

$$
\gamma(h)=\frac{1}{2 N(h)} \sum_{i=1}^{N(h)}\left[z\left(x_{i}\right)-z\left(x_{i}+h\right)\right]^{2},
$$

where $N(h)$ is the number of data pairs within a given lag distance $(h)$ and direction. $z\left(x_{i}\right)$ and $z\left(x_{i}+h\right)$ are sample elevations at location $\left(x_{i}\right)$ and $\left(x_{i}+h\right)$, respectively. If the values at $z\left(x_{i}\right)$ and $z\left(x_{i}+h\right)$ are autocorrelated, the result of equation (3) will be small, relative to an uncorrelated pair of points.

Empirical semivariogram was calculated by using all the pixels in a DEM of given cell size. The lag distance used was $1800 \mathrm{~m}$ and semivariance was calculated for the entire stretch of catchment (maximum distance). Moreover, the calculated semivariogram was a global one, i.e., a single semivariogram was computed for the entire catchment at a given DEM cell size. Subsequently, the calculated empirical semivariogram was fitted using spherical model (equation 4).

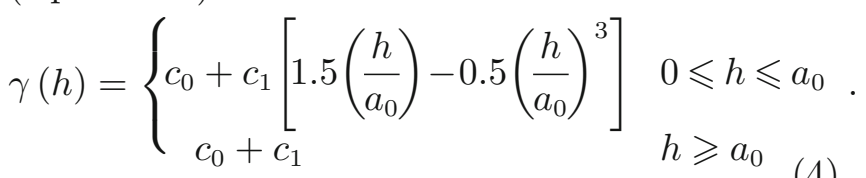


The semivariogram parameters that describe spatial structure of dataset are nugget $\left(c_{0}\right)$, sill $\left(c_{0}+c_{1}\right)$ and range $\left(a_{0}\right)$. Nugget, semivariance value at zero lag distance, indicates the measurement error and spatial variability within a cell. Sill is the semivariance value at maximum distance of spatial dependence (range) and corresponds to the variance of the raster data. Range is the value of lag distance $(h)$ when the semivariogam levels off and it indicates the distance beyond which data are not spatially autocorrelated. Structural variance $\left(c_{1}\right)$ is corresponding to total variance due to spatial dependencies within the dataset. For semivariance at a lag distance of one pixel, equation (3) was calculated for only one lag distance equivalent to one pixel size of different DEMs.

\section{Results and discussion}

\subsection{Sink analysis}

Although, the original contour lines were devoid of depression contour and TOPOGRID has an iterative smoothening algorithm to remove pits, still different amount of sinks were observed in the interpolated DEM surfaces. The number of sinks and the total surface area of the sinks were the parameters that were analyzed in sink analysis and the results are presented in figure 2 .

Figure 2 demonstrates a remarkable difference in distribution of sinks in the DEM surface of different resolutions. The number of sinks was found to be highest at $30 \mathrm{~m}$ resolution, which was observed to decrease linearly with increasing cell size. The $90 \mathrm{~m}$ resolution DEM has only 1954 sinks. The number of sinks alone may not fully reveal interpolation artifacts, hence the total surface area of sinks was also compared (figure 2). The total surface area of sinks (in hectare or the percentage of total area) was found to be much influenced by the cell resolution. In contrast to sink number, the surface area of sink was observed to increase exponentially with increasing cell size. For $30 \mathrm{~m}$ DEM the surface area of sink covers only $0.14 \%$ of total area, while for the $90 \mathrm{~m}$ DEM the sink surface area is about $0.56 \%$ of the total area. The effect of terrain complexity on the sink distribution was explored by plotting the sink density (number of sinks per $\mathrm{km}^{2}$ ) against the cell size for different terrain complexity classes (figure 3).

Figure 3 shows that the sink density is dependent on the terrain complexity. The TOPOGRID interpolator produced different extent of sinks for different terrain complexity classes. The value of sink density was found to be the highest in case of TCC-3 which is corresponding to the most complex terrain. On the other hand, sink density was lowest in case of TCC-1, which is corresponding to the least complex terrain of the study area. However, the magnitude of sink density in all the terrain complexity classes decreased with increasing cell size. It was also observed that the magnitude of sink density for all the terrain complexity classes merge to almost same value at $90 \mathrm{~m}$ resolution. This observation supports the fact that merging of smaller sinks to create larger ones is a more acceptable reason for decreased sink density with increasing cell size. Moreover, the results of sink analysis asserted that sink surface area should also be considered for a complete sink analysis.

\subsection{Fractal dimension}

In order to explore the artifacts in stream networks (model streams) extracted from DEMs of different resolution, fractal dimension was

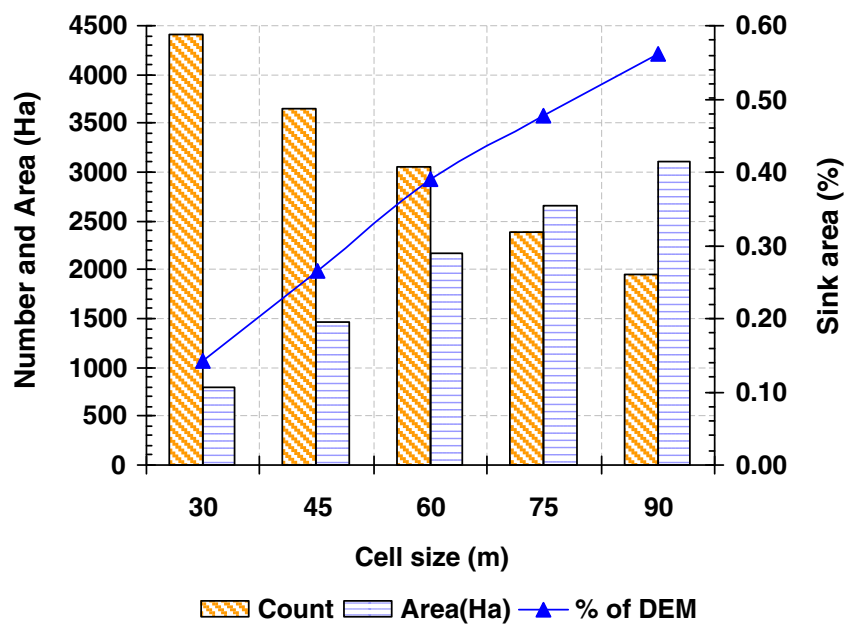

Figure 2. Number and total surface area (hectares and percentage) of sink at different cell size.

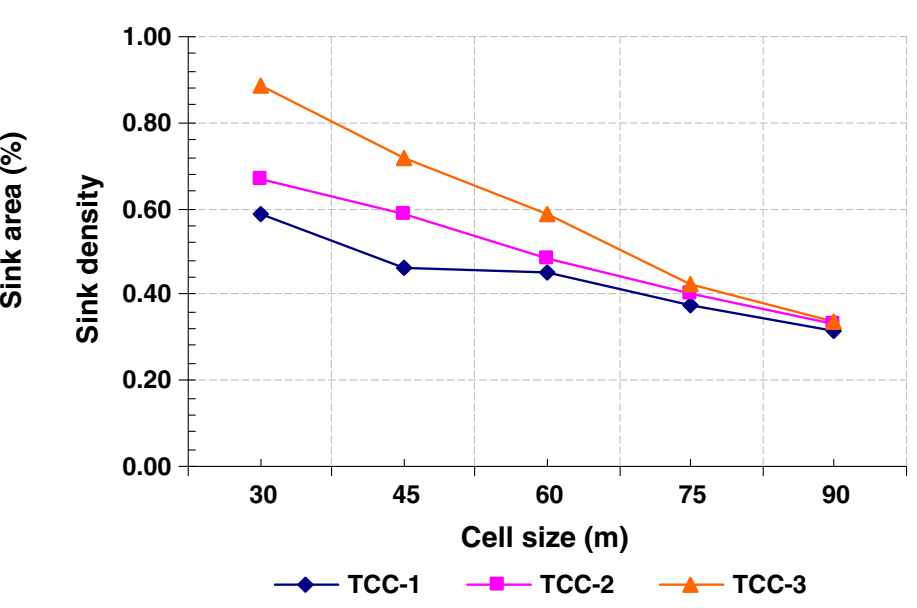

Figure 3. Sink density of DEMs at different cell size and terrain complexity classes. 
computed using box count method and compared to that of a reference stream network (figure 4).

Figure $4(\mathrm{~d})$ indicates that the fractal dimension value of the stream network derived from DEM of $90 \mathrm{~m}$ cell size is much higher than fractal dimension value computed for those derived from DEMs of other resolution and is closer to the fractal dimension value of reference stream (shown as straight line in the figure $4 \mathrm{~d}$ ). A higher value of fractal dimension for $90 \mathrm{~m}$ DEM indicates that the stream network produced from it has required degree of curvature leading to the true appearance of stream network. While the low fractal dimension value for DEMs of other resolutions may be due to relatively straight stream networks (stream feathering-artifact) which may in turn be due to more flat area. This is indicative of the fact that a $90 \mathrm{~m} \mathrm{DEM}$ is more accurate to derive the stream network with least artifact and most resemblance to the reference streams.

\subsection{Information content-entropy}

Entropy was calculated to estimate the change in the amount of information with the increase in cell size of the interpolated DEMs. The global entropy was calculated from the elevation value (in meter) up to two significant digits and the results are presented in table 1.

Table 1 demonstrates a decrease in entropy (ENT) with increasing cell size, as it was anticipated. But the calculation of entropy is known to be influenced by the number of pixels being used. The observed reduction in entropy may be due to the reduction in number of pixels (Stoy et al 2009). Hence, entropy of DEMs of different resolution

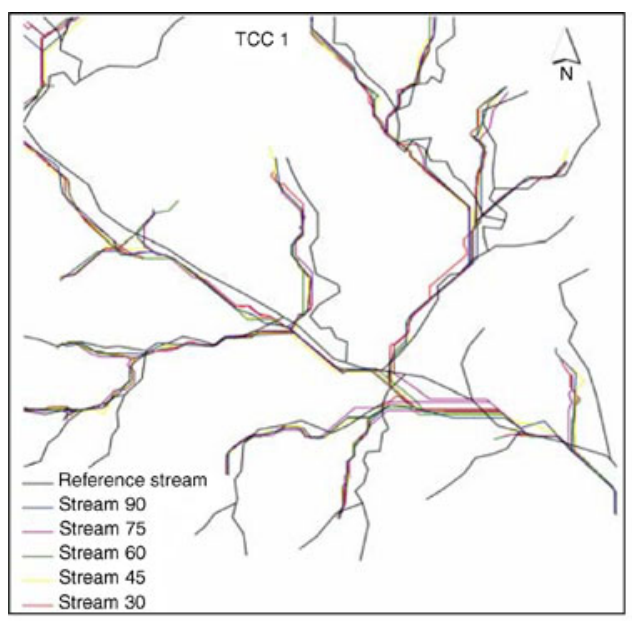

(a) TCC1

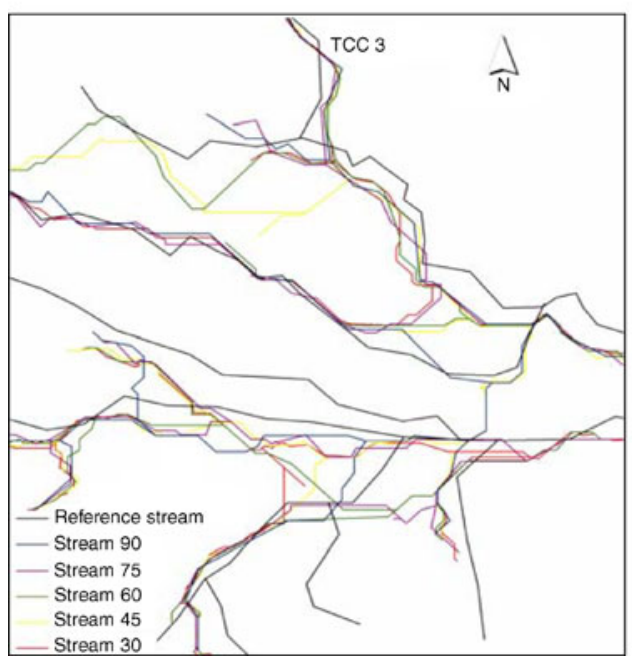

(c) TCC3

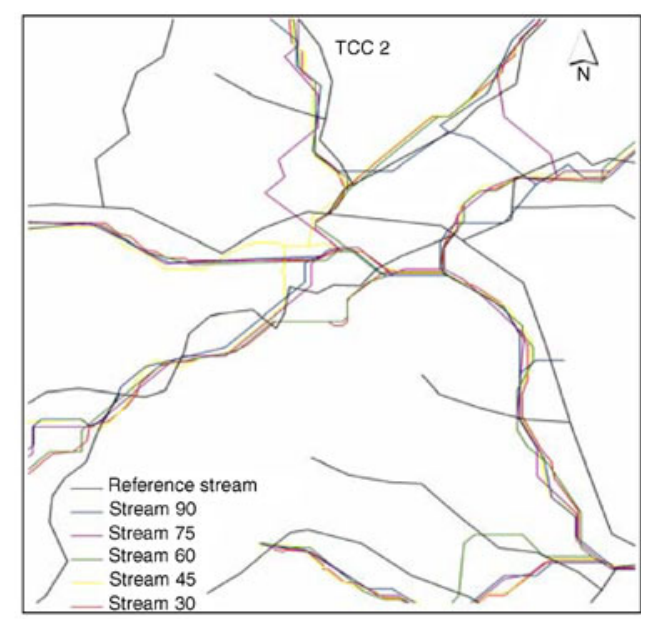

(b) TCC2

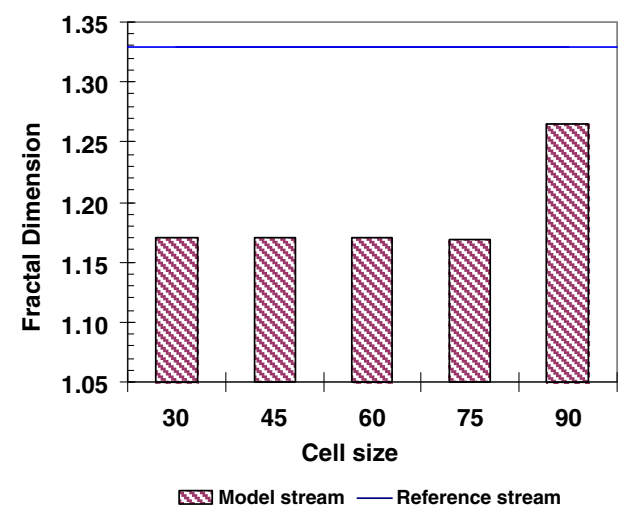

(d) Fractal dimension

Figure 4. Representative stream network delineated using DEM of different resolution in each TCC (a-c) and the values of fractal dimension for stream extracted from of different DEMs (d). 
Table 1. Global entropy of DEMs at different resolution.

\begin{tabular}{lcccc}
\hline $\begin{array}{l}\text { Cell size } \\
(\mathrm{m})\end{array}$ & Entropy & $\begin{array}{c}\text { Normalized } \\
\text { entropy }\end{array}$ & Change & \% Change \\
\hline 30 & 10.765 & 0.793 & - & - \\
45 & 10.733 & 0.834 & 0.041 & 5.16 \\
60 & 10.693 & 0.867 & 0.031 & 3.67 \\
75 & 10.680 & 0.891 & 0.027 & 3.09 \\
90 & 10.665 & 0.914 & 0.023 & 2.57 \\
\hline
\end{tabular}

cannot be compared until and unless the effect of number of elementary pixel is eliminated. Therefore, the calculated entropy values were normalized by dividing them with $2 \log _{e}(N)$, where $N$ is the total number of pixels in the DEM of respective cell size. The value of normalized entropy (NENT) presented in table 1 indicates a completely reversal of the non-normalized trend, i.e., increase in entropy with increasing cell size. However, the margin of increase in normalized entropy at a particular cell size from its preceding cell size gradually decreases from $45(5.16 \%)$ to $90 \mathrm{~m}(2.57 \%)$. The results suggest that a DEM of $90 \mathrm{~m}$ cell size is sufficient for representing the topographic heterogeneity of the study site. Cai and Wang (2006) also found that a $90 \mathrm{~m}$ DEM might be as good as a $30 \mathrm{~m}$ DEM for deriving TWI. The effect of terrain on information content was explored by calculating the mean local entropy $(900 \times 900 \mathrm{~m}$ window $)$ for each terrain complexity class and the results are shown in figure 5.

Figure 5 shows that among the different terrain complexity classes the mean local entropy value (normalized) increases with increasing terrain complexity at all the resolutions, i.e., the value of information content follows the order of TCC-3 $>$
TCC-2 > TCC-1. However, the value of entropy (actual) was found to be highest for TCC-2 despite its less spatial variability than TCC-3. The only reason for this discrepancy is that the TCC-2 covers the larger portion of the study site $(49.06 \%)$ as compared to TCC-3 (37.89\%) and TCC-1 (13.04\%) and hence has more number of elementary pixels at a particular resolution. This fact further strengthens the requirement of normalization of entropy to eliminate the effect of total number of elementary pixels used in entropy calculation. The mean local entropy (ENT) of DEM at different resolutions follow more or less similar trend as that of global entropy, i.e., decrease in entropy with increasing cell size for all the terrain complexity classes. However, the normalized entropy (NENT) showed a reverse trend, i.e., increase in information content with increasing cell size. It indicates that $90 \mathrm{~m}$ cell size some what corresponds to the information content of the original contour line used for interpolation. It was observed that there is no improvement in the information content of the interpolated DEMs on increasing the resolution beyond $90 \mathrm{~m}$ in any of the terrain complexity classes. It indicates that oversampling (small cell size) of actual elevation information of contour line leads to DEM

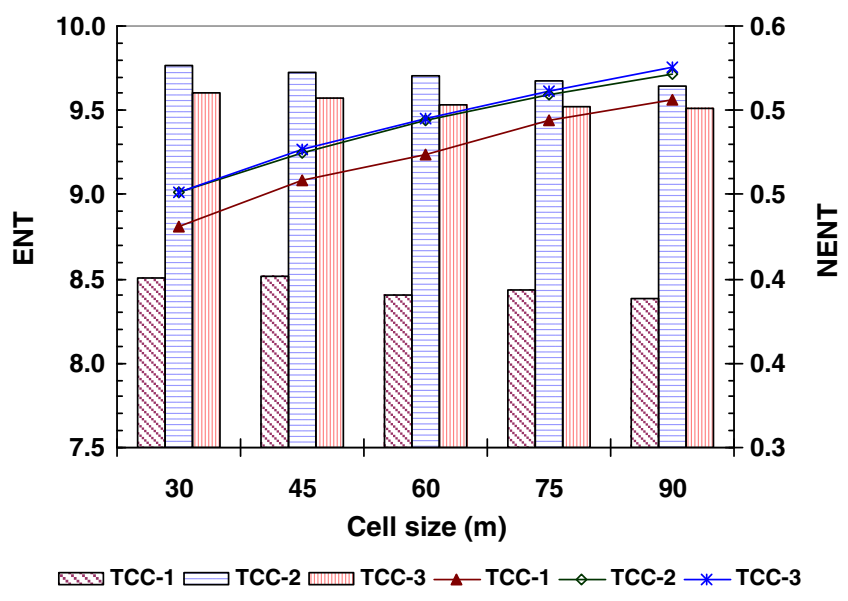

Figure 5. Mean local entropy of DEMs at different cell size and terrain complexity classes $(\mathrm{ENT}=$ entropy \& NENT $=$ normalized entropy).

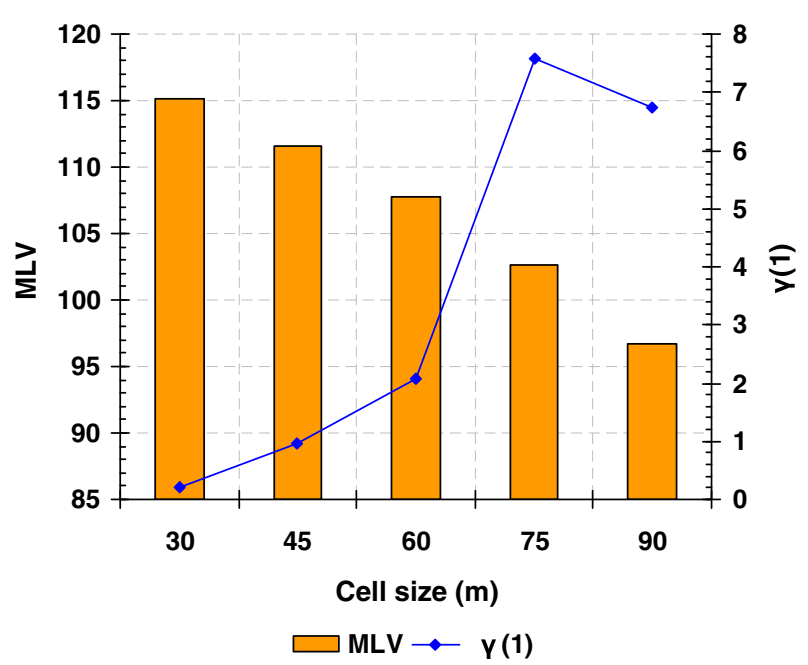

Figure 6. Mean local variance (MLV) and semivariance at a lag distance of one pixel $(\gamma(1))$ at different cell size. 


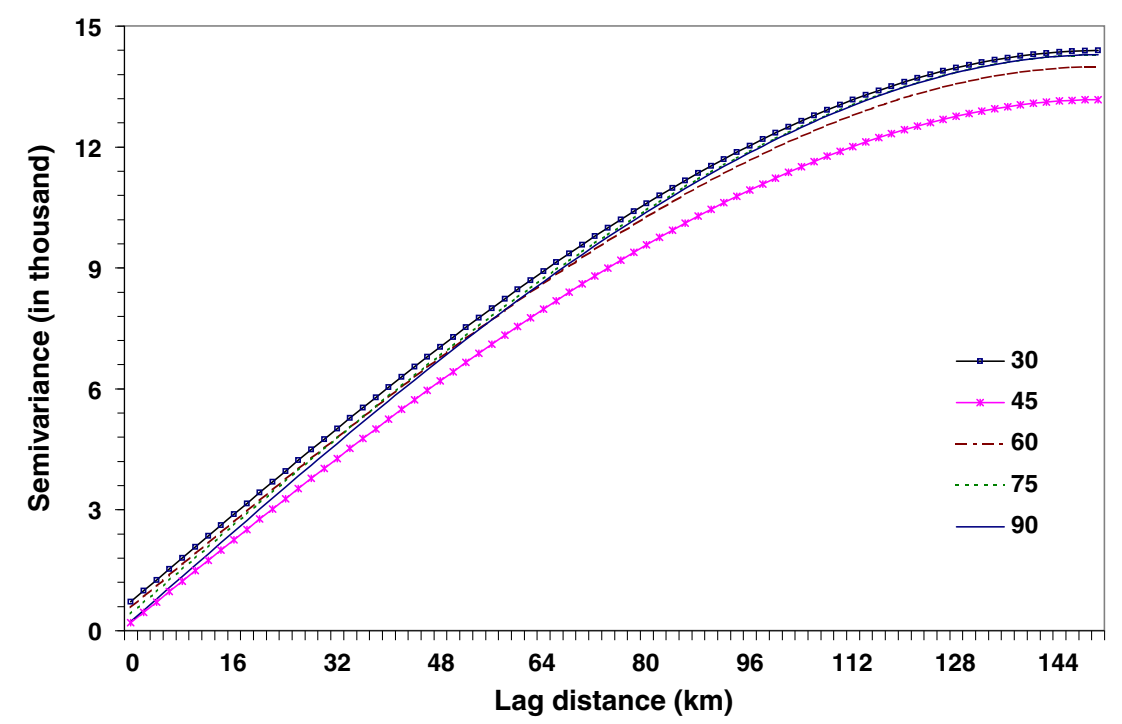

Figure 7. The modelled semivariograms (spherical) for elevation value of DEM of different resolutions.

surface with redundant information (flat area or uniform slope).

\subsection{Spatial variability - semivariogram modelling}

The MLV calculated using $900 \mathrm{~m}$ sliding window is presented in figure 6 . There is a steady decrease in MLV with increasing cell size due to high correlation between the neighbouring pixels. However, the calculation of semivariance at lag distance of one pixel or $\gamma(1)$ gives a contrasting result (figure 6).

The value of $\gamma(1)$ steadily increases from finer to coarser resolution DEM. The comparative low value of $\gamma(1)$ for high resolution DEM may be due to oversampling that leads to data redundancy in the form of flat area or area with insignificant slope. $\gamma(1)$, similar to MLV measures the mean local spatial variability considering the spatial structure of raster data which is lacking in case of MLV. Hence, $\gamma(1)$ appears to be more appropriate measure of variability than MLV.

The detailed spatial variability structures of DEM of different resolutions were simulated using variogram modelling. Omni-directional semivariograms with same lag size $(2000 \mathrm{~m})$ were calculated for the entire extent of the study area and fitted using spherical model (figure 7).

Figure 7 demonstrates that modelled semivariogram from DEMs of different cell sizes are similar in shape. Although, the range value of all the semivariograms appear to be nearly equal to their nugget and sill value differ considerably from each other. The corresponding model parameters like nugget, sill and range are presented in table 2 .

When the experimental semivariograms were calculated using raster data, the nugget variance $\left(C_{0}\right)$ implies a noise term, that is, measurement error variance and within-cell variability. The sill variance $(C)$ implies the variance of the raster data. The nugget variance and sill variance generally decrease as the cell spacing increases. The range parameter $\left(A_{1}\right)$ indicates the distance beyond which spatial dependence among the elevation data points disappears, and it tends to increase as the cell spacing increases.

It is evident from table 2 that the $C_{0}$ decreases with change in spacing from finer to coarser, and the only exception was observed at $60 \mathrm{~m}$ where the $C_{0}$ is higher than the preceding value. Higher values of nugget were observed at low cell size. But it

Table 2. Variogram parameters of DEM fitted with spherical model.

\begin{tabular}{lccccc}
\hline $\begin{array}{l}\text { Cell size } \\
(\mathrm{m})\end{array}$ & $C_{0}$ & $C_{1}$ & $C=C_{0}+C_{1}$ & $C_{0} / C$ & $\begin{array}{c}A_{1} \\
\text { (meter) }\end{array}$ \\
\hline 30 & 711.25 & 13681.75 & 14393.00 & 0.05 & 150440 \\
45 & 191.52 & 12980.48 & 13172.00 & 0.01 & 150470 \\
60 & 573.08 & 13412.92 & 13986.00 & 0.04 & 150480 \\
75 & 418.45 & 13870.55 & 14289.00 & 0.03 & 150470 \\
90 & 222.97 & 14066.03 & 14289.00 & 0.02 & 150500 \\
\hline
\end{tabular}

$C_{0}$ - nugget, $C_{1}$ - model structure variance, $C$ - sill, $A_{1}$ - range. 
has also associated noise or measurement error that needs to be separated. The value of sill $(C)$ (the semivariance value at which the variogram levels off) corresponds to the total variance of the raster data and it increased with change in resolution from finer to coarser. The only exception observed was the $45 \mathrm{~m}$ resolution DEM, for which value of $C$ was less than its corresponding value at $30 \mathrm{~m}$ resolution. The structural variance $\left(C_{1}\right)$ which corresponds to the variance due to autocorrelation in the sample data can be obtained by subtracting the nugget value from its corresponding sill. For $C_{1}$, a trend similar to sill was observed. The above observation indicates that the high resolution (small cell size) DEM has high noise (as observed in nugget value) and the actual spatial variability is less when compared to DEMs of higher grid cell size. Moreover, the value of nugget/sill ratio $\left(C_{0} / C\right)$ was used to explain the amount of spatial variability that cannot be explained by the geostatistical model. The value of $\left(C_{0} / C\right)$ was found to be highest $(0.05)$ for $30 \mathrm{~m}$ cell size DEM while it is lowest for $45 \mathrm{~m}$ resolution DEM (0.01) followed by $90 \mathrm{~m}$ cell size DEM (0.02). It indicates that $5 \%$ and $2 \%$ of the total spatial variability of the $30 \mathrm{~m}$ and $90 \mathrm{~m}$ cell size DEMs respectively are unexplained variability, i.e., due to random noise. Since nugget/sill ratio defines the proportion of the variability that occurs at short distances (shorter than the smallest sampling interval) a small value usually indicates that higher accuracy can be obtained for a given sampling density of elevation value. The range parameter $\left(A_{1}\right)$ implies the distance beyond which spatial dependence disappears or there is no spatial autocorrelation among data points. In this study, the value of range increases with DEM resolution becoming coarser, with only deviations observed at $75 \mathrm{~m}$. This increasing trend in range value may be due to loss of micro scale variation (nugget), which leads to increased spatial autocorrelation in the neighbourhood.

\section{Conclusion}

This study presented four simple methods which may serve as guide to select an optimum DEM cell size objectively in prior to detailed hydrological modelling. The interpolation artifact in DEM surface and extracted stream network were explored through sink analysis and fractal dimension respectively. The $90 \mathrm{~m}$ resolution DEM had the fewest total number of sinks. Sink density decreased in each TCC strata as coarseness increased to $90 \mathrm{~m}$. The fractal dimension computed on stream networks derived from the interpolated DEMs of $90 \mathrm{~m}$ grid cell resolutions best approximated the computed fractal dimension for the reference stream network. The information content of DEM surface measured using both local and global entropy indicated that the DEM of $90 \mathrm{~m}$ resolution had highest degree of information and was corresponding to information content of the original contour lines used for DEM interpolation. Spatial variability of interpolated DEM surfaces was investigated using semivariogram modelling which revealed that spatial variability of the DEM surface increased from 30 to $90 \mathrm{~m}$ resolution DEM while the random noise content decreased from fine to coarse resolution DEM over the same resolutions. All these analyses enabled us to identify the appropriate cell size objectively based on intrinsic quality of DEM surface and avoided the chance of oversampling or undersampling. The results indicated that a DEM of $90 \mathrm{~m}$ cell size is sufficient for capturing the terrain variability of the study area and for subsequent hydrologic modelling.

\section{Acknowledgements}

Authors are thankful to the Head, Agricultural \& Food Engineering Department for providing all necessary laboratory facilities to carry out this research work. Authors also duly acknowledge the critical suggestions provided by the Associate Editor and the anonymous reviewers.

\section{References}

Abedini M J and Shaghaghian M R 2009 Exploring scaling laws in surface topography; Chaos, Solitons \&6 Fractals 42(4) 2373-2383.

Aryal S K and Bates B C 2008 Effects of catchment discretization on topographic index distributions; J. Hydrol. 359(1-2) 150-163.

Atkinson P M and Curran P J 1995 Defining an optimal size of support for remote sensing investigations; IEEE Trans. Geosci. Remote Sens. 33(3) 768-776.

Cai X and Wang D 2006 Spatial autocorrelation of topographic index in catchments; J. Hydrol. 328(3-4) 581-591.

Chaubey I, Cotter A S, Costello T A and Soerens T S 2005 Effect of DEM data resolution on SWAT output uncertainty; Hydrol. Process. 19(3) 621-628.

Chen Q and Gong P 2004 Automatic variogram parameter extraction for textural classification of the panchromatic IKONOS imagery; IEEE Trans. Geosci. Remote Sens. 42(5) 1106-15.

Dutta D and Nakayama K 2009 Effects of spatial grid resolution on river flow and surface inundation simulation by physically based distributed modelling approach; Hydrol. Process. 23(4) 534-545.

Florinsky I V and Kuryakova G A 2000 Determination of grid size for digital terrain modelling in landscape investigations - Exemplified by soil moisture distribution at a micro-scale; Int. J. Geogra. Inf. Sci. 14(8) 815-832.

Goovaerts P 1999 Geostatistics in soil science: State-of-theart and perspectives; Geoderma 89 1-45. 
Hancock G R 2005 The use of digital elevation models in the identification and characterization of catchments over different grid scales; Hydrol. Process. 19(9) 1727-1749.

Hengl T 2006 Finding the right pixel size; Comput. Geosci. 32(9) $1283-1298$.

Kuo W L, Steenhuis T S, McCulloch C E, Mohler C L, Weinstein D A, DeGloria S D and Swaney D P 1999 Effect of grid size on runoff and soil moisture for a variablesource-area hydrology model; Water Resour. Res. 35(11) 3419-3428.

Lacroix M P, Martz L W, Kite G W and Garbrecht J 2002 Using digital terrain analysis modeling techniques for the parameterization of a hydrologic model; Environmental Modelling and Software 17(2) 127-136.

Lee G S and Lee K H 2006 Scaling effect for estimating soil loss in the RUSLE model using remotely sensed geospatial data in Korea; Hydrol. Earth Syst. Sci. Discuss. 3(1) $135-157$.

Liu X, Wang Y and Jin B 2009 Determination of suitable cell size for grid based Digital Elevation Model, Proceeding of SPIE International Symposium on Spatial Analysis, Spatial-Temporal Data Modeling, and Data Mining, 13-14 October 2009, Wuhan, China.

Mandelbrot B 1982 The fractal geometry of nature; Freeman, San Francisco, 460 p.

Rojas R, Velleux M, Julien P Y and Johnson B E 2008 Grid scale effects on watershed soil erosion models; J. Hydrol. Eng. 13(9) 793-802.

Shannon C and Weaver W 1949 The mathematical theory of communication; University of Illinois Press, Urbana.

Sharma A, Tiwari K N and Bhadoria P B S 2009 Measuring the accuracy of contour interpolated digital elevation models; J. Indian Soc. Remote Sens. 37(1) 139-146.
Singh R, Tiwari K N and Mal B C 2006 Hydrological studies for small watershed in India using the ANSWERS model; J. Hydrol. 318(1-4) 184-199.

Stoy P C, Williams M, Spadavecchia L, Bell R A, PrietoBlanco A, Evans J G and van Wijk M T 2009 Using information theory to determine optimum pixel size and shape for ecological studies: Aggregating land surface characteristics in arctic ecosystems; Ecosystems 12(4) $574-589$.

Sun W, Xu G, Gong P and Liang S 2006 Fractal analysis of remotely sensed images: A review of methods and applications; Int. J. Remote Sens. 27(20) 4963-4990.

Sun L Q, Hu C and Chen G 2008 Effects of DEM resolution on the TOPMODEL; Shuikexue Jinzhan/Advances in Water Science 19(5) 699-706.

Thompson J A, Bell J C and Butler C A 2001 Digital elevation model resolution: Effects on terrain attribute calculation and quantitative soil-landscape modeling; Geoderma 100(1-2) 67-89.

Wechsler S P 2007 Uncertainties associated with digital elevation models for hydrologic applications: A review; Hydrol. Earth Syst. Sci. 11 1481-1500.

Wilson J P and Gallant J C 2000 Digital terrain analysis; In: Terrain Analysis: Principles and Applications (eds) Wilson J P and Gallant G C, John Wiley \& Sons, pp. 1-27.

Wu S, Li J and Huang G 2005 An evaluation of grid size uncertainty in empirical soil loss modeling with digital elevation models; Environ. Model. Assess. 10(1) 33-42.

Zhang J X, Chang K T and Wu J Q 2008 Effects of DEM Resolution and Source on Soil Erosion Modeling: a Case Study Using the WEPP Model; Int. J. Geogr. Inf. Sci. 22(8) 925-942. 